\title{
Self-Assembly of Plasmonic Near-Perfect Absorbers of Light: The Effect of Particle Size
}

\author{
Gus O. Bonin, ${ }^{\dagger}$ Steven J. Barrow, ${ }^{\dagger}$ Timothy U. Connell, ${ }^{\dagger}$ Ann Roberts, ${ }^{\ddagger}$ Anthony S. R. \\ Chesman, ${ }^{*}, \uparrow, \S$ and Daniel E. Gómez*, ${ }^{*}$ \\ tSchool of Science, RMIT University, Melbourne, VIC 3000, Australia \\ $¥$ ARC Centre of Excellence for Transformative Meta-Optical Systems, School of Physics, The University of \\ Melbourne, VIC 3010, Australia \\ ICSIRO Manufacturing, Clayton, VIC 3169, Australia \\ \&Melbourne Centre for Nanofabrication, Clayton, VIC 3169, Australia \\ E-mail: anthony.chesman@csiro.au; daniel.gomez@rmit.edu.au
}




\begin{abstract}
Structures capable of perfect light absorption promise technological advancements in varied applications, including sensing, opto-electronics and photo-catalysis. While it is possible to realise such structures by placing a monolayer of metal nanostructures above a reflecting surface, there remains limited studies on what effect particle size plays on their capacity to absorb light. Here we fabricate near-perfect absorbers using colloidal Au nanoparticles, via their electrostatic self-assembly on a $\mathrm{TiO}_{2}$ film supported by a gold mirror. This method enables the control of interparticle spacing, thus minimizing reflection to achieve optimal absorption. Slightly altering the nanoparticle size in these structures reveals significant changes in the spectral separation of hybrid optical modes. We rationalize this observation by interpreting data with a coupled-mode theory that provides a thorough basis for creating functional absorbers using complex colloids and outlines the key considerations for achieving a broadened spectral response.
\end{abstract}

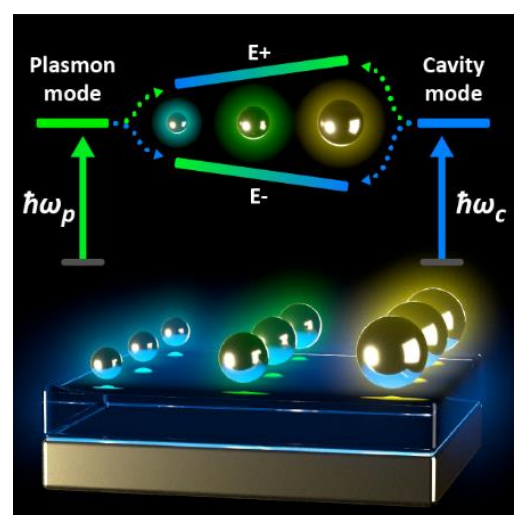


Efficient absorption of light is a fundamental property underlying a variety of fields, including: energy harvesting, ${ }^{1}$ bio-medical sensing, ${ }^{2-3}$ surface imaging ${ }^{4}$ and light guiding ${ }^{5,6} \mathrm{It}$ is possible to create surfaces that exhibit perfect or near-complete absorption of light, including plasmonic near-perfect absorbers, optical analogues of the Salisbury screen. ${ }^{7}$ Plasmonic absorbers achieve complete absorption of light due to destructive interference between all scattering pathways, resulting in energy absorption and dissipation in metallic nanostructures. Plasmonic absorbers typically comprise three layers: a mirror, a spacer and a nanostructured surface, and support both localised surface plasmons and Fabry-Pérot resonances (Figure 1). The mutual interaction of these resonances with incident light also leads to the formation of strongly coupled plasmon-cavity hybrid states. ${ }^{8}$

The conditions required for achieving perfect absorption of light at particular frequencies can be achieved through fine-tuning the geometry and the composition of the metal nanoparticles, as well as the thickness and refractive index of the spacer layer. ${ }^{9-10}$ Top-down nanofabrication methods, such as electron beam lithography, offer exquisite control of the particle size, shape and arrangement but are limited to relatively small surface areas. Alternatively, direct deposition and subsequent thermal annealing of thin metal films results in dense nanoparticle monolayers but does not allow precise control of the nanoparticle geometry. Electrostatic self-assembly of colloids onto surfaces, where nanoparticles synthesized by wet chemical methods are deposited as monolayers, shows great promise as a technique to optimize both perfect absorption and strong coupling of the plasmonic and Fabry-Pérot resonant modes. This colloid selfassembly strategy addresses the limitations of physical deposition techniques and allows precise control of nanoparticle size, shape, spacing and composition, including the possibility of using bi-metallic nanostructures. ${ }^{8,11-14}$

In this study, we develop and demonstrate an electrostatic self-assembly strategy to deposit a monolayer of chemically-synthesized gold nanoparticles on top of a mirror-spacer to create scalable plasmonic near-perfect absorbers. The subsequent control of nanoparticle geometry and inter-particle spacing allows for a systematic study of the effect of nanoparticle size on the optical properties of plasmonic near-perfect absorbers. We show that the plasmon-cavity coupling strength is proportional to nanoparticle size. Quantitative analysis using a coupled-mode theory allowed us to ascertain that particle size affects the damping rates, which in turn modifies the interaction between localised surface plasmons, Fabry-Pérot cavity resonances, and incident light. This work is organized in two parts. Firstly, we detail the development of the electrostatic self-assembly of chemically-synthesised metal nanoparticles. In the second part we analyse the optical properties of the resulting plasmonic near-perfect absorbers using coupled-mode theory.

Electrostatic self-assembly. For this study, the substrates comprised an Au mirror $(100 \mathrm{~nm})$ and a thin $\mathrm{TiO}_{2}$ dielectric film ( $35 \mathrm{~nm}$ ), both deposited on a silicon support via electron beam evaporation. Citrate-capped Au nanoparticles were synthesized according to a previously reported procedure. ${ }^{15}$ The uniform deposition of Au nanoparticles was achieved using a four step self-assembly procedure: (1) acidic pre-treatment of the $\mathrm{TiO}_{2}$ surface; (2) increasing the $\mathrm{pH}$ of Au colloids through addition of $\mathrm{NaOH}$; (3) drop casting the colloidal solution onto the substrate prior to (4) washing and drying the substrate surface (Figure 1A).

In step (1), mirror-spacer substrates were treated with an acidic solution ( $0.1 \mathrm{M}$ aqueous $\mathrm{HCl}$ ) well below the isoelectric point of $\mathrm{TiO}_{2}(\mathrm{pH} 6) .{ }^{16}$ This treatment alters the charge and chemistry of the surface, inducing positive charge and removing carbon residue. ${ }^{17}$ Rinsing and drying the substrates prior to self-assembly were critical for ensuring good nanoparticle monolayers, as Au nanoparticles are known to melt at low $\mathrm{pH}^{18}$ particularly over the timescales necessary to achieve dense surface coverage. Self-assembly of citratecapped Au nanoparticles (steps (2) and (3)) is driven by two electrostatic interactions: (i) attraction between the negatively charged particles and the positively charged substrate and (ii) the repulsion of like-charged 
particles in solution (Figure 1A). A concentrated colloidal solution was drop-cast onto the substrate surface, applying as much solution as surface tension allowed, and then left undisturbed for three hours before rinsing. By fine-tuning the interplay of the nanoparticle-substrate attraction and inter-nanoparticle repulsion, we achieved a uniform, close-packed nanoparticle assembly, as evidenced by scanning electron microscopy (Figure 1B). This self-assembly strategy was limited only by substrate size and is easily scaled to an area of several $\mathrm{cm}^{2}$.
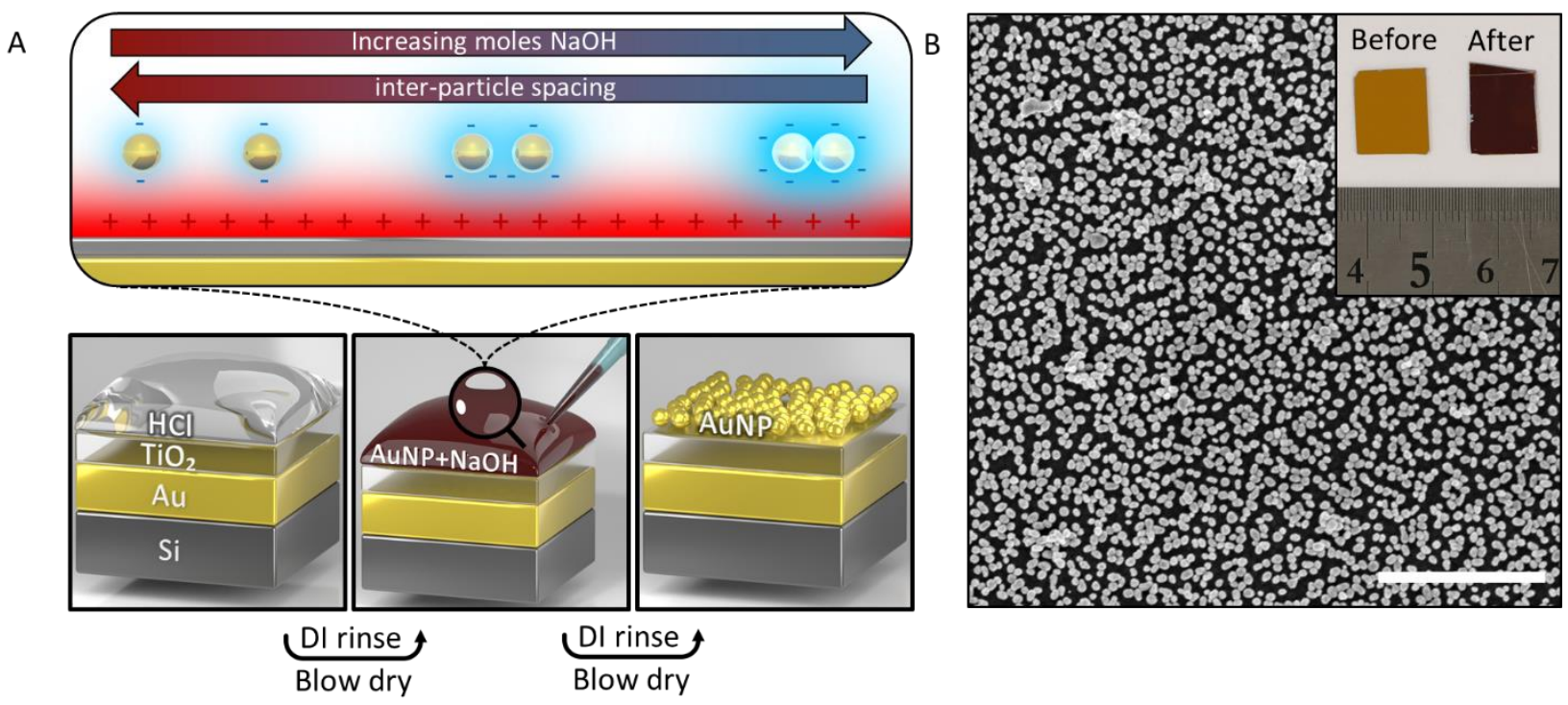

Figure 1: Mechanism of Self-assembly. A) Step-by-step procedure of electrostatic self-assembly detailing the governing mechanisms, namely attraction between the negatively charged particles and positively charged substrate and the repulsion of like-charged particles in solution. Blue (red) shading denotes negative (positive) charge. B) A representative SEM image (scalebar represents $1 \mu \mathrm{m}$ ) of the resulting nanoparticle monolayer formed from electrostatic self-assembly and (inset, scale in $\mathrm{cm}$ ) image of samples before and after monolayer electrostatic self-assembly.

It was observed that the interparticle spacing decreased with increasing $\mathrm{pH}$, and highly concentrated nanoparticle solutions proved much more effective at reducing the interparticle spacing as they are easier to destabilize. Importantly, the high pH of this solution, well above the isoelectric point of the dielectric spacer layer, helps prevent the deposition of multiple nanoparticle layers, as substrate-nanoparticle repulsion gradually increases during assembly. While the addition of $\mathrm{NaOH}$ increases the negative charge of the Au nanoparticles by deprotonating the citrate capping ligands, ${ }^{19}$ the increased concentration of sodium ions ultimately reduces the electrostatic repulsion between nanoparticles. Consequently, colloidal Au nanoparticles (diameter $47 \mathrm{~nm}$ ) are brought to the cusp of aggregation via the addition of aqueous $\mathrm{NaOH}$, with $2.5 \mu \mathrm{M} \mathrm{NaOH}$ in the final colloidal solution generating the most uniform monolayers (Figure 2A). Image analysis of scanning electron microscope (SEM) images revealed that surface coverage increased from $30-88 \%$ as the $[\mathrm{NaOH}]$ was increased. Samples with higher surface coverage (> 60\%) exhibited significant amounts of high-order aggregates.

Increasing surface coverage also impacted the ability of the structure to absorb light. Optical absorption (A) spectra were calculated from experimental transmission $(T)$ and reflectance $(R)$ measurements and consisted of multiple overlapping bands (Figure 2B), originating from the interaction of surface plasmon and Fabry-Pérot resonances, in addition to the presence of the $d$ band transition of Au. Sharp peaks and the highest absorption values ( $A_{\max }>97 \%$ ) occurred when nanoparticle surface coverage remained below $50 \%$. Near-perfect absorbers that displayed significant aggregation by microscopy (samples with $77 \%$ and $88 \%$ surface coverage) exhibited decreased absorption maxima and concomitant increases in spectral linewidths. We attribute this absorption decrease to increased scattering from aggregates. Achieving 
maximum absorption is therefore a fine balance of decreasing interparticle spacing without inducing nanoparticle aggregation.

A
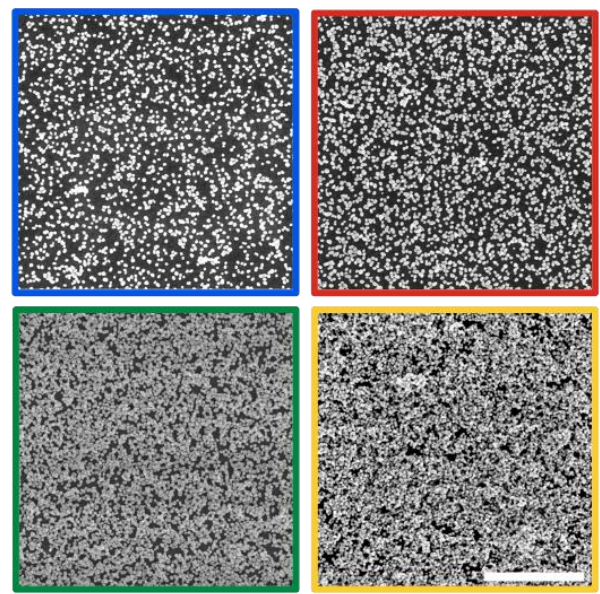

B

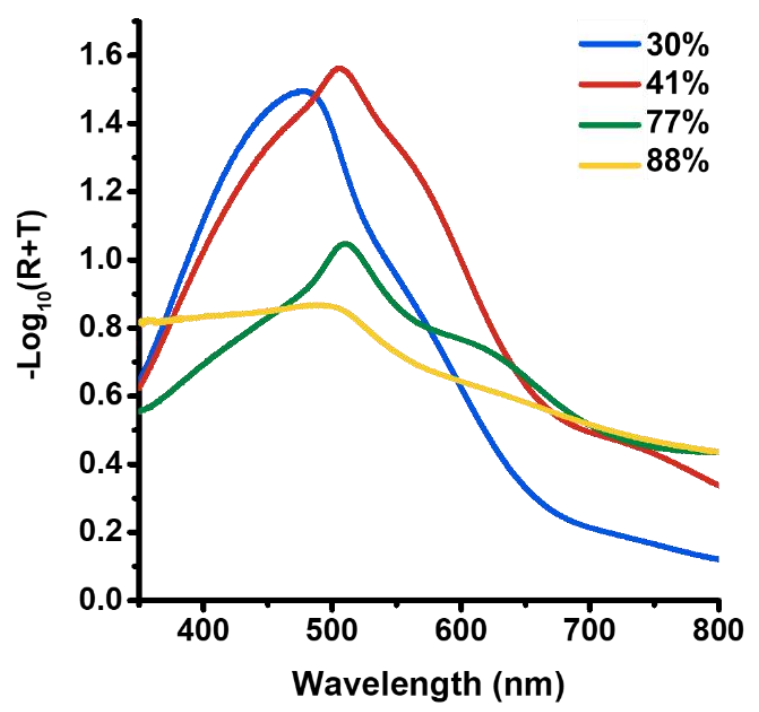

Figure 2: Effect of inter-particle spacing on absorption. A) Representative SEM images of plasmonic near-perfect absorbers with self-assembled monolayers of $47 \mathrm{~nm}$ citrate-capped Au nanoparticles. The colloidal solutions contained $2.0 \mu \mathrm{M}$ (blue), $2.5 \mu \mathrm{M}$ (red), $3.0 \mu \mathrm{M}$ (green) and $3.5 \mu \mathrm{M}$ (yellow) $\mathrm{NaOH}$. B) Absorption spectra of plasmonic near-perfect absorbers with differing surface area coverage.

The resonant frequency of the Fabry-Pérot cavity depends on the spacer thickness and its refractive index (see Supporting Information, Figure S1). When this frequency is matched to the local surface plasmon resonance of the nanoparticles, strong coupling can take place, resulting in hybrid plasmon-cavity states that manifest experimentally as spectral doublets. ${ }^{9-10}$ In order to study the effect of particle size on the optical properties of plasmonic near-perfect absorbers of light, we chose sub-wavelength particle sizes, for which the frequency of the localised surface plasmon resonance is nearly size-independent, but which exhibit different scattering and absorption cross-sections. ${ }^{9-10}$ The optical absorption spectra of plasmonic near-perfect absorbers with different sized nanoparticles, but otherwise identically prepared, all exhibited two overlapping bands and absorption maxima indicative of near-perfect absorption $\left(A_{\max }>90 \%\right)$ at the resonant frequency (Figure $3 A$ ). Representative SEM images revealed only minor changes in surface coverage, which we attribute to the difference in varying particle size (Figure 3B). As nanoparticle size 
increased from 47, 54 and $58 \mathrm{~nm}$, the position and relative separation of the measured absorption doublets changed [the high energy peak remained relatively constant (510, 528 and $526 \mathrm{~nm}$ ) whilst the other bathochromically shifted $(565,600$ and $642 \mathrm{~nm})$, respectively]. A dramatic peak separation is observed in comparison to the small changes in local surface plasmon resonance attributed to the varying particle size (black dotted lines). Statistical analysis between each particle size distribution revealed (at the 0.05 confidence level) all distributions were significantly different (Figure S2). To explain the observed increased of peak separation with particle size, we attempt to employ a coupled oscillator model.

A

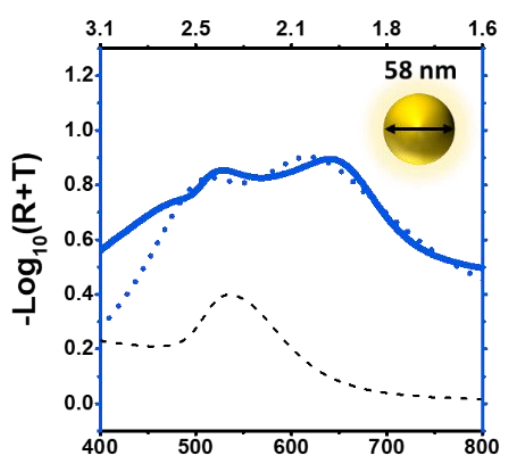

B

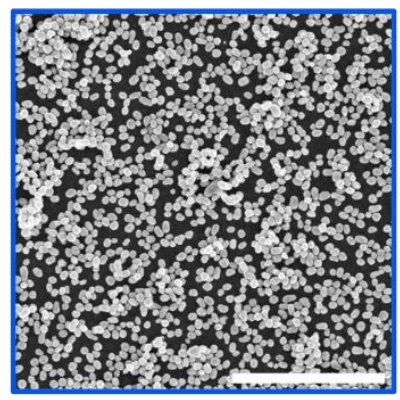

$56 \%$

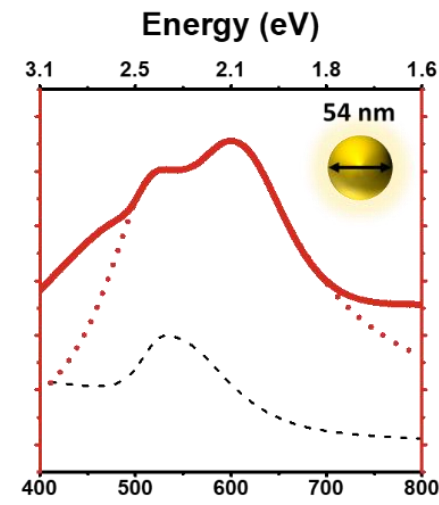

Wavelength $(\mathrm{nm})$

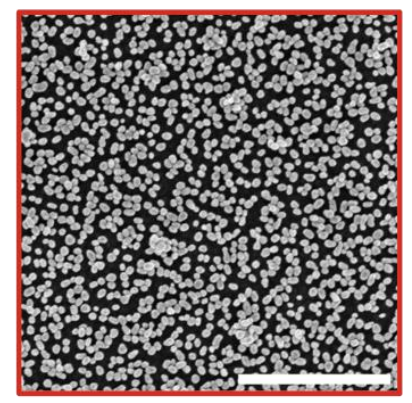

$49 \%$
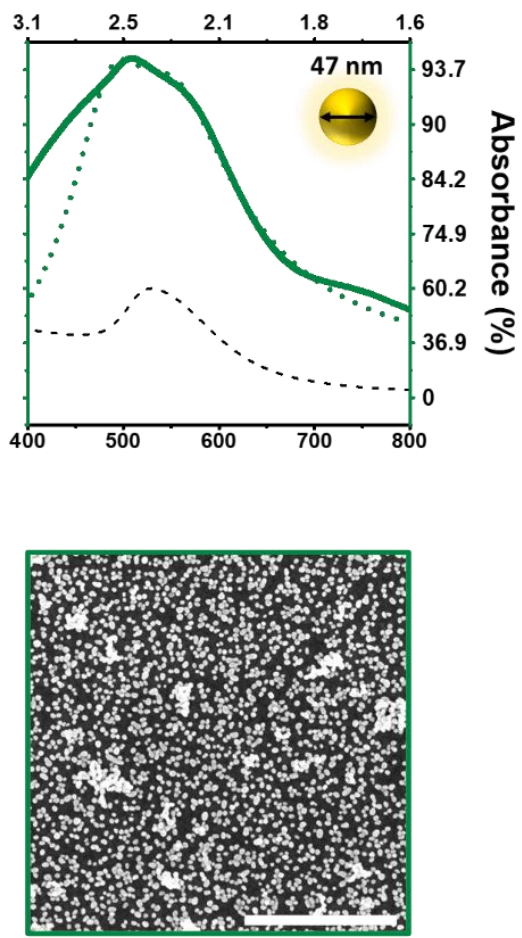

$39 \%$

Figure 3: Effect of particle size on absorption. A) Optical absorption spectra (solid line) and calculated absorption (dashed line) for plasmonic near-perfect absorbers made with Au nanoparticles of different sizes. Black dotted lines correspond to normalised absorption of the corresponding Au colloids, indicating their respective local surface plasmon resonance. B) Corresponding SEM images (scalebar represents $1 \mu \mathrm{m}$ ).

Strong coupling between two resonant systems is often described using an effective Hamiltonian (Figure 4A). The two systems (characterised by the complex resonant energies $E_{p}-i \gamma_{p}$ and $E_{c}-i \gamma_{c}$ ) can reversibly exchange energy via a mechanism accounted for by the coupling constant $\Omega\left(\gamma_{p, c}\right.$ quantifies the non-radiative losses associated with each system). This interaction generates two new hybridised resonances $^{11} E_{ \pm}$given by: $E_{ \pm}=\left[E_{p}+E_{c}-i\left(\gamma_{p}+\gamma_{c}\right)\right] / 2 \pm \sqrt{\Omega^{2}+\left[E_{c}-E_{p}-i\left(\gamma_{p}-\gamma_{c}\right)\right]^{2} / 4}$, which implies that when the system is in resonance (i.e. when $\mathrm{E}_{\mathrm{p}}=\mathrm{E}_{\mathrm{c}}$ ) doublets are observed only when $\sqrt{4 \Omega^{2}-\left(\gamma_{p}-\gamma_{c}\right)^{2}}>\left(\gamma_{p}+\gamma_{c}\right)$. Careful control of the $\mathrm{TiO}_{2}$ spacer thickness (19-44 $\left.\mathrm{nm}\right)$ found perfect agreement between measured absorption maxima (corresponding to $E_{ \pm}$) and this model for a coupling constant $\Omega \sim 320 \mathrm{meV} .{ }^{9}$ The same model, however, seemingly fails to account for our experimental observations (Figure 3A). Despite a fixed $\mathrm{TiO}_{2}$ thickness (and therefore a fixed Fabry-Pérot resonance $\left.E_{c}\right)$ and a size-independent localised surface plasmon resonance frequency $\left(E_{p}\right.$, dotted lines Figures 3A, S3), the measured splitting $E_{+}-E_{-}$increases with nanoparticle size, giving 282, 277 and 396 
meV for diameters of 47,54 and $58 \mathrm{~nm}$, respectively. To interpret this result, we developed a coupledmode theory analysis of these structures that allows us to simultaneously account for the observed spectral doublets and high absorption of incident electromagnetic energy.

Coupled-mode theory ${ }^{20-21}$. This theory is an approximate, insightful and often accurate representation of electromagnetic oscillation and wave propagation in a coupled system, which can be traced back to an eigenmode expansion of Maxwell's equations for a coupled system. Within this theory, the absorber consists of two resonant subsystems that reversibly exchange energy at a rate $\Omega$ (Figure 4B). As in the coupled-oscillator model, the only adjustable parameters, other than $\Omega$, are the resonant energies ( $\mathrm{E}_{\mathrm{p}}$ and $\left.E_{c}\right)$ and their corresponding rates $\left(\gamma_{p}\right.$ and $\left.\gamma_{c}\right)$. Importantly, we assume that only the nanoparticle monolayer interacts strongly with incident radiation, whereas the Fabry-Pérot resonant mode does not, meaning that the corresponding rate $\gamma_{\mathrm{p}}$ now comprises both radiative and non-radiative components (i.e. $\gamma_{p}=\gamma_{p r}+\gamma_{p n r}$, Figure 4B). Because our near-perfect absorbers vary only in nanoparticle size, we further assume that the cavity parameters remained fixed across all samples: $\gamma_{c}=269 \mathrm{meV}$ and $\mathrm{E}_{\mathrm{c}}=2.46 \mathrm{eV}$.

According to coupled-mode theory, ${ }^{22-23}$ the amount of power stored in the localised surface plasmon resonance is expressed by a quantity $|p|^{2}$, whereas the one stored in the Fabry-Pérot resonance is given by $|c|^{2}$. The amplitude of $p$ evolves in time according to:

$$
\frac{d p}{d t}=\left(i \omega_{p}-\gamma_{p}\right) p+i \Omega c+s_{+} \sqrt{2 \gamma_{p r}}
$$

where $\omega_{p}$ is the plasmon resonance frequency $\left(E_{p}=\hbar \omega_{p}\right)$. For the particle sizes that we have employed in our study, this resonance frequency is size-independent (see black lines in Figure $3 \mathrm{~A}$ ). ${ }^{24}$ The final term of this equation accounts for the power $\left|s_{+}\right|^{2}$ supplied by external incident illumination, which couples radiatively to the particle plasmon resonance.

Metal nanoparticles also interact (with Rabi frequency $\Omega$ ) with the Fabry-Pérot cavity mode (resonance frequency $\omega_{c}$ ), whose amplitude $c$ evolves in time as:

$$
\frac{d c}{d t}=\left(i \omega_{c}-\gamma_{c}\right) c+i \Omega p
$$

The cavity damping rate $\gamma_{c}$ is purely non-radiative and originates from structural imperfections that allow the escape or scattering of light by the cavity. $\omega_{c}$ is the resonance frequency of the Fabry-Pérot mode, which in turn depends on parameters such as the thickness and refractive index of the spacer layer (Figure 1), and the magnitude of the phase shifts experienced by light as it reflects from the mirror and nanoparticle monolayer.

The plasmon-cavity system couples energy into outgoing waves according to:

$$
s_{-}=-s_{+}+p \sqrt{2 \gamma_{p r}}
$$

which enables the definition of an amplitude reflection coefficient $r=s_{-} / s_{+}$. Equation (3) corresponds to a single port for energy exchange between light and the system. This differs from the case of Coherent Perfect Absorption, where two ports (incident beams) with well-defined phase differences are considered. ${ }^{25}$ Finally, assuming a time-harmonic incident wave of frequency $\omega$ [i.e. $s_{+}=s_{+}(\omega) e^{i \omega t}$ ], direct integration of the coupled Equations (1) and (2) and their substitution into Equation (3) leads to the amplitude reflection coefficient $r$ : 


$$
r=-1+\frac{2 \mathrm{i} \gamma_{\mathrm{pr}}\left(\omega-\omega_{c}-\mathrm{i} \gamma_{c}\right)}{\Omega^{2}+\left(\mathrm{i} \gamma_{c}+\omega_{c}-\omega\right)\left[\omega-\omega_{p}-\mathrm{i}\left(\gamma_{p n r}+\gamma_{p r}\right)\right]}
$$

There are two situations where perfect absorption (i.e. $r=0$ ) of light can take place (found by solving for the frequencies at which Equation (4) equals zero). The first situation occurs when the plasmon and cavity mode are resonant (i.e. $\omega_{p}=\omega_{c}$ ) and the radiative decay rate of the plasmonic sub-system $\gamma_{p r}$ matches the total non-radiative decay rate of the system: $\gamma_{p r}=\gamma_{c}+\gamma_{p n r}$ (equivalently, $\gamma_{c c}$ in Figure 4C). Under this so called critical coupling condition, perfect absorption can be observed in two spectral bands that are separated in energy by an amount $2 \hbar \sqrt{\Omega^{2}-\gamma_{c}^{2}}$, requiring that $\Omega^{2}>\gamma_{c}^{2}$ to clearly discern the two absorption bands. The second condition for perfect absorption to occur is whenever the radiative rate $\gamma_{p r}=\Omega^{2} / \gamma_{c}+\gamma_{p n r}$, resulting in a single absorption maximum (also referred to as the weak critical coupling condition (or $\gamma_{w c c}$ in Figure $4 \mathrm{C}$ ). ${ }^{25}$

A

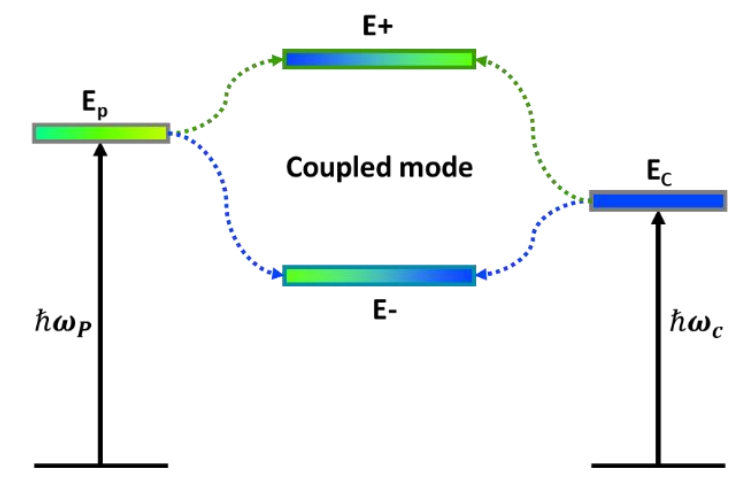

C

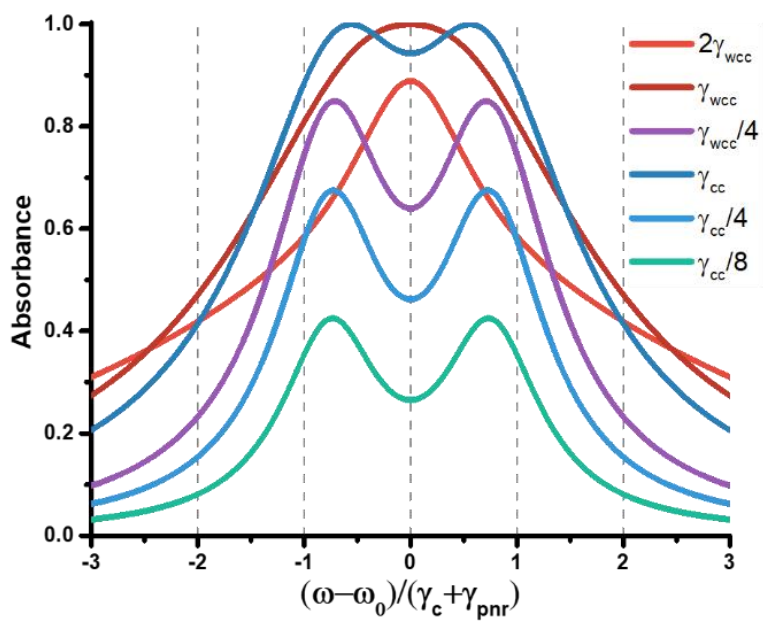

E
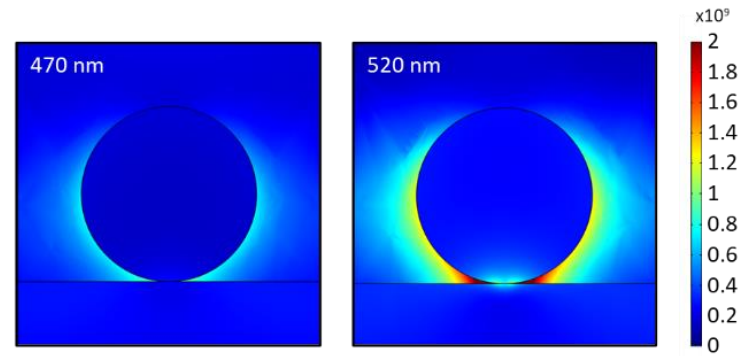

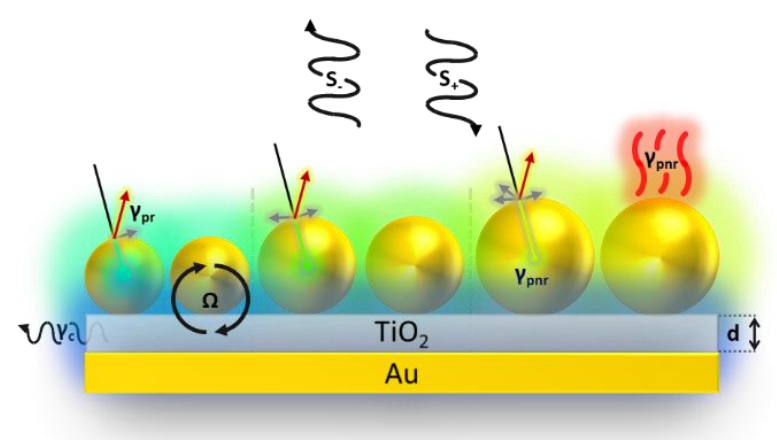

D

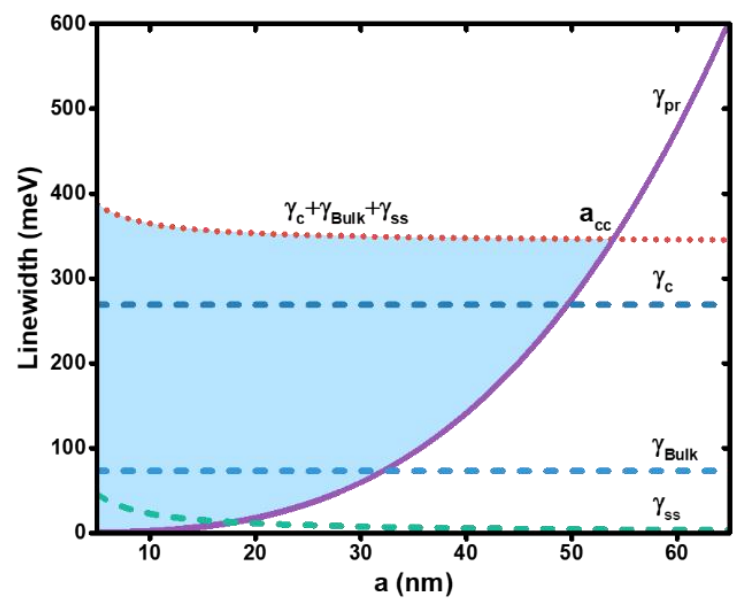

F

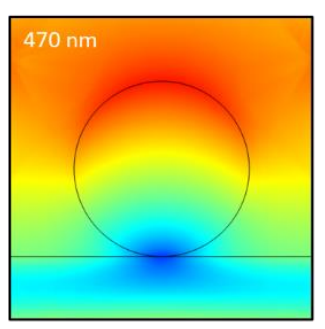

Figure 4: The effects of nanoparticle scattering and absorption on coupling strength. A) Energy level diagram of the components in a near-perfect absorber contributing to the observed strong coupling between the $\mathrm{Au}$ nanoparticle monolayer and the $\mathrm{TiO}_{2}$ spacer layer. B) Representative diagram of the plasmonic near-perfect absorber's interaction with light according to coupled-mode theory. C) Theoretical model displaying the absorption capabilities and peak-splitting characteristics from varying degrees of strong 
or weak critical coupling $\left(\gamma_{c c}\right.$ and $\gamma_{w c c}$, respectively). D) Plasmon linewidth $v s$ particle radius (a). The linewidth is decomposed into its constituents $\gamma_{p}=\gamma_{B u l k}+\gamma_{s s}+\gamma_{p r}$, where $\gamma_{B u l k}$ is the "bulk" contribution, $\gamma_{s s}$ is the surface scattering, $\gamma_{p r}$ the radiative damping and $\gamma_{c}$ is the cavity damping rate from Equation (2). $a_{c c}$ corresponds to a size that satisfies the critical coupling condition. Cross sectional maps of the electromagnetic fields corresponding to the magnitude of $\mathbf{E}$ ) the electric field and $\mathbf{F}$ ) the magnetic field at wavelengths corresponding to peak absorption for a particle of diameter $58 \mathrm{~nm}$ at the wavelengths indicated.

According to these predictions, systematic increases in the radiative rate $\gamma_{p r}$ of the plasmon sub-system could enable a transition to critical coupling (and consequent perfect absorption, Figure 4C). For a sufficiently large value of the coupling constant $\Omega$, the measured optical absorption spectrum should consist of a doublet, with absorption maxima that reach unity whenever $\gamma_{p r}=\gamma_{c c}$ (Figure 4C). Increasing this rate past this point will transition the system into the weak critical coupling condition $\left(\gamma_{p r}=\gamma_{w c c}\right)$, whereby the spectral doublet coalesces into a single band exhibiting unit absorbance (Figure 4C). Further increases in $\gamma_{p r}$ will ultimately lead to reduced light absorption.

This model fully accounts for the photophysics of a plasmonic near-perfect absorber. We have modelled our experimental data using Equation (4), resulting in the dashed lines shown in Figure 3A and the obtained fit parameters of Table 1 . These results indicate that the plasmon resonance radiative and non-radiative decay rates, in addition to the plasmon-cavity coupling $\Omega$, increase with nanoparticle size. There are two factors that contribute to a variation in the radiative and non-radiative decay rates of the localized surface plasmon resonance. Firstly, due to mechanisms such as surface scattering, inter-band ( $d$-sp) excitation and radiative decay damping, the decay rates of the localized surface plasmon resonance intrinsically depend on nanoparticle size. ${ }^{26,27}$ The second factor is the interaction between metal nanoparticles and their mirror images: a radiating dipole (e.g. a plasmonic nanoparticle) placed above a reflecting interface at a particular distance can exhibit an increased decay rate..$^{28-29}$ The interaction between nanoparticles and their mirror images re-normalizes their polarizability, which is partly manifested in modifications to the particle plasmon resonance linewidth. ${ }^{28}$ These interactions depend on the separation distance between the nanoparticle and the reflecting surface, and it is important to bear in mind that this distance also controls the cavity resonance frequency $\omega_{c}$, leading to a complex interplay of parameters that dictate the optical properties of the system, as encapsulated in Equation (4).

Table 1. Parameters of the best fit of the data of Figure 3 to the coupled-mode theory. $2 a$ is the particle diameter, $\nu_{c}$ is the cavity damping rate, $\nu_{r}$ is the radiative damping rate, $\nu_{n r}$ is the non-radiative damping rate, $\Omega$ is the coupling constant (Rates expressed in equivalent energy units). $E_{c}$ is the resonant energy of the cavity and $E_{p}$ is the resonant energy of the particles.

\begin{tabular}{ccccccc}
\hline \hline $2 \mathrm{a}(\mathrm{nm})$ & $\gamma_{\mathrm{c}}(\mathrm{eV})$ & $\gamma_{\mathrm{r}}(\mathrm{eV})$ & $\gamma_{\mathrm{nr}}(\mathrm{eV})$ & $\Omega(\mathrm{eV})$ & $E_{c}(\mathrm{eV})$ & $E_{p}(\mathrm{eV})$ \\
\hline 47 & 0.269 & 0.278 & 0.326 & 0.199 & 2.30 & 2.35 \\
54 & 0.269 & 0.271 & 0.359 & 0.204 & 2.25 & 2.25 \\
58 & 0.269 & 0.319 & 0.554 & 0.217 & 2.25 & 2.25 \\
\hline \hline
\end{tabular}

The decay rate of the localized surface plasmon resonance of small spheres can be approximately written as a function of particle radius $a$, according to $\gamma_{p}(a)=\gamma_{B u l k}+\frac{3 A v_{F}}{4 a}+\frac{4}{3} h \kappa a^{3}$, where the first term is the size-independent "bulk" term ( $\gamma_{B u l k} \sim 73 \mathrm{meV}$ in the spectral range of interest here) which accounts for intrinsic damping mechanisms in the bulk metal. ${ }^{27}$ The second term corresponds to the surface scattering contribution, which is due to inelastic collisions that shorten the electron mean free path for finite-sized nanoparticles $\left(v_{F}\right.$ is the Fermi velocity, which for $A u$ is $1.4 \mathrm{~nm} / \mathrm{fs}^{30}$ and $\left.A=0.33\right) .{ }^{27}$ The last contribution 
corresponds to radiative damping, which originates from the capacity of a nanoparticle to radiate light due to the dipole induced when these are irradiated ( $h$ is Planck's constant and $\left.\kappa=4 \times 10^{-7} \mathrm{fs}^{-1} \mathrm{~nm}^{-3}\right) \cdot{ }^{26}$ Figure 4D shows all of these components as a function of particle size, not accounting for the effect of the mirror on the total decay rate. The blue-shaded area of Figure 4D represents where particles are below the critical coupling condition, which is in turn reached when the radiative rate $\left(\gamma_{p r}\right.$, purple line) intercepts the total non-radiative decay rate of the system $\left(\gamma_{c}+\gamma_{B u l k}+\frac{3 A v_{F}}{4 a}\right.$, red dotted line). This means that for a fixed distance $d$ away from the mirror, the specific nanoparticle size $a_{c c}$ represents where perfect absorption of light can occur. Away from this size, the system will not exhibit perfect absorbance as seen in Figure 4C. The magnitude of this critical particle size $\left(a_{c c}\right)$ is in turn dependent on the separation distance $(d)$ from the mirror $a_{c c}=a_{c c}(d)$, as the interaction of the particle with the retarded field due to the presence of the mirror can modify the total decay rate, ${ }^{31}$ shifting the position of this critical size. It is important to note that this simple analysis is valid for small particle sizes (i.e. when the effects of higher-order multipoles and retardation can be ignored) and for individual particles. When the inter-particle distance is smaller than the incident wavelength, as in our monolayers, the entire film acts as a uniform layer for which the resonant frequency shifts due to inter-particle interactions. ${ }^{32}$

The model reasonably accounts for our experimental observation that peak splitting $\left(\Delta E=E_{+}-E_{-}\right)$ increases with nanoparticle size (Figure $3 \mathrm{~A}$ ). Within an electrostatic limit (valid here due to sub-wavelength nanoparticle size), the interaction of the nanoparticles with radiation is dominated by a dipole coupling term $\Omega \sim \mathbf{p} \cdot \mathbf{E}$, which involves the dipole moment $\mathbf{p}$ of the nanoparticle's localised surface plasmon resonance and the electric field $\mathbf{E}$ surrounding the particles. The cavity parameters remain fixed in all the structures of Figure $\mathbf{3 A}$, so it is valid to assume that the (evanescent) electric field $\mathbf{E}$ of the Fabry-Pérot resonance also remains constant. The magnitude of the dipole moment $\mathbf{p}$ instead increases with particle volume, ${ }^{33}$ resulting in the positive correlation between $\Omega$ and nanoparticle size, and a consequent increase in $\Delta \mathrm{E}=2 \hbar \sqrt{\Omega^{2}-\gamma_{c}^{2}}$. The fit parameters of Table 1 indicate that our structures are not operating in a strict strong coupling regime (since $\Omega \sim \gamma_{c}$ ), however, spectral doublets are clearly visible in Figure 3 .

Consequently, particle size affects both the ability to attain the critical coupling condition and the magnitude of the particle-cavity coupling strength $\Omega$. One implication of our analysis is that perfect absorption $(A=100 \%)$ may never be attainable in such experimental systems. Nanoparticle monolayers prepared via colloid self-assembly will always comprise a distribution of particle geometries (size, shape, Figure S2), which therefore imply a distribution of radiative rates $\gamma_{p r}$. While a subset of the particle population can attain critical coupling (i.e. $\gamma_{p r}=\gamma_{c}+\gamma_{p n r}$ ), there will always be other subsets within the population where the radiative rate either exceeds or falls short of the coupling criterion. However, as evidenced by our experimental results, near-perfect absorption (arbitrarily defined as $A>90 \%$ ) is possible for geometrically polydisperse nanoparticle ensembles.

The formation of plasmon-cavity hybrid states implies that incident energy on metal-dielectric-metal NP structures of the type outlined in Figure $\mathbf{1}$ is redistributed in the form of superpositions of plasmon-like and cavity-like excitations. This redistribution of energy can be visualised by calculating maps of the electromagnetic fields, obtained through a full-wave, finite element method solution of Maxwell equations, as implemented in COMSOL Multiphysics (Figures 4E, F simulation details in the Methods section of the Supporting Information). According to these calculations, the observed spectral doublets correspond to a situation where the energy is predominantly confined either as a pseudo-particle plasmon resonance, observed at a wavelength of $520 \mathrm{~nm}$ (close to $E_{-}$), or as a kind of Fabry-Pérot mode of the structure, seen more predominantly at $470 \mathrm{~nm}$ (close to $\mathrm{E}_{+}$) (Figure 4A, Figure $\mathbf{5 4}$ ). 
In summary, we have demonstrated the effect of nanoparticle size on the optical properties of plasmonic near-perfect absorbers. The observed optical absorbance and increase in hybrid mode separation with nanoparticle size were well described using a coupled-mode theory which fully accounts for the interplay of energy between the plasmon, Fabry-Perot cavity, and incident light. For a given nanoparticle size, nearperfect absorption of light is achieved whenever the radiative damping rate matches the total non-radiative decay rate in the system. The radiative rate, in addition to being controlled by particle size, can also be modulated by altering the distance between particle and mirror. This understanding grants flexibility and control over the spectral response of absorbing materials, and outlines the key parameters required to achieve high optical absorption and strong cavity-plasmon coupling. The study was enabled by the development of an electrostatic self-assembly protocol, achieved by balancing electrostatic repulsion and attraction forces to drive the formation of a densely packed nanoparticle monolayer. This solution based process for the formation of nanoparticle monolayers opens the prospect for exploring the fabrication of plasmonic near-perfect absorbers of light with complex colloids, including particles with sharp, nanoscale

corners which exhibit strong localisation of electromagnetic fields, nanoparticle "nanolenses" ${ }^{34}$ and nanoparticle alloys known to be efficient catalysts.

\section{Experimental methods}

Detailed procedures are provided in the Supporting Information.

We acknowledge the efforts of William Durkacz for their continued assistance and direction with the 3D art associated with this work. We acknowledge Michael Wilms for insightful discussion. This work was performed in part at the Melbourne Centre for Nanofabrication (MCN) in the Victorian Node of the Australian National Fabrication Facility (ANFF). This work was performed in part at the RMIT Micro Nano Research Facility (MNRF) in the Victorian Node of the Australian National Fabrication Facility (ANFF). The authors acknowledge the facilities, and the scientific and technical assistance, of the RMIT Microscopy \& Microanalysis Research Facility at RMIT University. S.J.B thanks RMIT University for support through a ViceChancellor's Postdoctoral Fellowship. D.E.G. acknowledges the ARC for support (FT140100514). A.R. acknowledges the ARC for support (DP160100983). 


\section{References}

1. Peña-Rodríguez, O.; Prada, A.; Olivares, J.; Oliver, A.; Rodríguez-Fernández, L.; Silva-Pereyra, H. G.; Bringa, E.; Perlado, J. M.; Rivera, A. Understanding the ion-induced elongation of silver nanoparticles embedded in silica. Scientific Reports 2017, 7 (1), 922.

2. Muradov, N. Z. CO2-Free Production of Hydrogen by Catalytic Pyrolysis of Hydrocarbon Fuel. Energy \& Fuels 1998, 12 (1), 41-48.

3. Halas, N. J.; Lal, S.; Chang, W.-S.; Link, S.; Nordlander, P. Plasmons in Strongly Coupled Metallic Nanostructures. Chem. Rev. 2011, 111 (6), 3913-3961.

4. $\quad \mathrm{Xu}, \mathrm{H}$.; Aizpurua, J.; Käll, M.; Apell, P. Electromagnetic contributions to single-molecule sensitivity in surface-enhanced Raman scattering. Physical Review E 2000, 62 (3), 4318-4324.

5. $\quad$ Fang, Y.; Sun, M. Nanoplasmonic waveguides: towards applications in integrated nanophotonic circuits. Light: Science \& Applications 2015, 4 (6), e294-e294.

6. Pelton, M. Modified spontaneous emission in nanophotonic structures. Nature Photonics 2015, 9 (7), 427-435.

7. Salisbury, W. W. Absorbent body for electromagnetic waves. 1952.

8. Shi, Q.; Connell, T. U.; Xiao, Q.; Chesman, A. S. R.; Cheng, W.; Roberts, A.; Davis, T. J.; Gómez, D. E. Plasmene Metasurface Absorbers: Electromagnetic Hot Spots and Hot Carriers. ACS Photonics 2019, 6 (2), 314-321.

9. Shi, X.; Ueno, K.; Oshikiri, T.; Sun, Q.; Sasaki, K.; Misawa, H. Enhanced water splitting under modal strong coupling conditions. Nature Nanotechnology 2018, 13 (10), 953-958.

10. Ng, C.; Wesemann, L.; Panchenko, E.; Song, J.; Davis, T. J.; Roberts, A.; Gómez, D. E. Plasmonic Near-Complete Optical Absorption and Its Applications. Advanced Optical Materials 2019, $7(14), 1801660$.

11. Funston, A. M.; Novo, C.; Davis, T. J.; Mulvaney, P. Plasmon Coupling of Gold Nanorods at Short Distances and in Different Geometries. Nano Letters 2009, 9 (4), 1651-1658.

12. Chen, H.; Sun, Z.; Ni, W.; Woo, K. C.; Lin, H.-Q.; Sun, L.; Yan, C.; Wang, J. Plasmon Coupling in Clusters Composed of Two-Dimensionally Ordered Gold Nanocubes. Small 2009, 5 (18), 2111-2119.

13. Tabor, C.; Van Haute, D.; El-Sayed, M. A. Effect of Orientation on Plasmonic Coupling between Gold Nanorods. ACS Nano 2009, 3 (11), 3670-3678.

14. Zheng, Y.; Rosa, L.; Thai, T.; Ng, S. H.; Gómez, D. E.; Ohshima, H.; Bach, U. Asymmetric gold nanodimer arrays: electrostatic self-assembly and SERS activity. Journal of Materials Chemistry $A$ 2015, 3 (1), 240-249.

15. Kimling, J.; Maier, M.; Okenve, B.; Kotaidis, V.; Ballot, H.; Plech, A. Turkevich Method for Gold Nanoparticle Synthesis Revisited. The Journal of Physical Chemistry B 2006, 110 (32), 1570015707.

16. Kosmulski, M. Isoelectric points and points of zero charge of metal (hydr)oxides: 50years after Parks' review. Adv. Colloid Interface Sci. 2016, 238, 1-61.

17. Yu, J. C.; Yu, J.; Zhao, J. Enhanced photocatalytic activity of mesoporous and ordinary TiO2 thin films by sulfuric acid treatment. Applied Catalysis B: Environmental 2002, 36 (1), 31-43.

18. Reineck, P.; Lee, G. P.; Brick, D.; Karg, M.; Mulvaney, P.; Bach, U. A Solid-State Plasmonic Solar Cell via Metal Nanoparticle Self-Assembly. Advanced Materials 2012, 24 (35), 4750-4755.

19. Contreras-Trigo, B.; Díaz-García, V.; Guzmán-Gutierrez, E.; Sanhueza, I.; Coelho, P.; Godoy, S. E.; Torres, S.; Oyarzún, P. Slight pH Fluctuations in the Gold Nanoparticle Synthesis Process Influence the Performance of the Citrate Reduction Method. Sensors 2018, 18 (7), 2246.

20. Fan, S.; Suh, W.; Joannopoulos, J. D. Temporal coupled-mode theory for the Fano resonance in optical resonators. J. Opt. Soc. Am. A 2003, 20 (3), 569-572.

21. Zanotto, S.; Tredicucci, A. Universal lineshapes at the crossover between weak and strong critical coupling in Fano-resonant coupled oscillators. Scientific Reports 2016, 6 (1), 24592.

22. Mayer, M.; Schnepf, M. J.; König, T. A. F.; Fery, A. Colloidal Self-Assembly Concepts for Plasmonic Metasurfaces. Advanced Optical Materials 2019, 7 (1), 1800564. 
23. Brasse, Y.; Ng, C.; Magnozzi, M.; Zhang, H.; Mulvaney, P.; Fery, A.; Gómez, D. E. A Tunable Polymer-Metal Based Anti-Reflective Metasurface. Macromol. Rapid Commun. 2020, 41 (1), 1900415.

24. Davis, T. J.; Gómez, D. E. Colloquium: An algebraic model of localized surface plasmons and their interactions. Reviews of Modern Physics 2017, 89 (1), 011003.

25. Zanotto, S.; Mezzapesa, F. P.; Bianco, F.; Biasiol, G.; Baldacci, L.; Vitiello, M. S.; Sorba, L.; Colombelli, R.; Tredicucci, A. Perfect energy-feeding into strongly coupled systems and interferometric control of polariton absorption. Nature Physics 2014, 10 (11), 830-834.

26. Sönnichsen, C.; Franzl, T.; Wilk, T.; von Plessen, G.; Feldmann, J.; Wilson, O.; Mulvaney, P. Drastic Reduction of Plasmon Damping in Gold Nanorods. Phys. Rev. Lett. 2002, 88 (7), 077402.

27. Novo, C.; Gomez, D.; Perez-Juste, J.; Zhang, Z.; Petrova, H.; Reismann, M.; Mulvaney, P.; Hartland, G. V. Contributions from radiation damping and surface scattering to the linewidth of the longitudinal plasmon band of gold nanorods: a single particle study. Physical Chemistry Chemical Physics 2006, 8 (30), 3540-3546.

28. Kwadrin, A.; Osorio, C. I.; Koenderink, A. F. Backaction in metasurface etalons. Physical Review B 2016, 93 (10), 104301.

29. Chance, R. R., Prock, A. and Silbey, R. Molecular Fluorescence and Energy Transfer Near Interfaces. In Adv. Chem. Phys., 2007; pp 1-65.

30. Berciaud, S.; Cognet, L.; Tamarat, P.; Lounis, B. Observation of Intrinsic Size Effects in the Optical Response of Individual Gold Nanoparticles. Nano Letters 2005, 5 (3), 515-518.

31. R. R. Chance, A. P., R. Silbey. Molecular Fluorescence and Energy Transfer Near Interfaces. In Adv. Chem. Phys., 1978; pp 1-65.

32. Yu, R.; Mazumder, P.; Borrelli, N. F.; Carrilero, A.; Ghosh, D. S.; Maniyara, R. A.; Baker, D.; García de Abajo, F. J.; Pruneri, V. Structural Coloring of Glass Using Dewetted Nanoparticles and Ultrathin Films of Metals. ACS Photonics 2016, 3 (7), 1194-1201.

33. Craig F. Bohren, D. R. H. Particles Small Compared with the Wavelength. In Absorption and Scattering of Light by Small Particles, 1998; pp 130-157.

34. Lloyd, J. A.; Ng, S. H.; Liu, A. C. Y.; Zhu, Y.; Chao, W.; Coenen, T.; Etheridge, J.; Gómez, D. E.; Bach, U. Plasmonic Nanolenses: Electrostatic Self-Assembly of Hierarchical Nanoparticle Trimers and Their Response to Optical and Electron Beam Stimuli. ACS Nano 2017, 11 (2), 1604-1612. 


\section{University Library}

\section{- M M N E R VA A gateway to Melbourne's research publications}

Minerva Access is the Institutional Repository of The University of Melbourne

Author/s:

Bonin, GO;Barrow, SJ;Connell, TU;Roberts, A;Chesman, ASR;Gomez, DE

Title:

Self-Assembly of Plasmonic Near-Perfect Absorbers of Light: The Effect of Particle Size

Date:

2020-10-01

Citation:

Bonin, G. O., Barrow, S. J., Connell, T. U., Roberts, A., Chesman, A. S. R. \& Gomez, D. E. (2020). Self-Assembly of Plasmonic Near-Perfect Absorbers of Light: The Effect of Particle Size. JOURNAL OF PHYSICAL CHEMISTRY LETTERS, 11 (19), pp.8378-8385. https:// doi.org/10.1021/acs.jpclett.0c02461.

Persistent Link:

http://hdl.handle.net/11343/268134 